\title{
DISTRIBUTION OF FREE FLIGKT TIMES IN A HARD SPHERE GAS
}

\author{
F.W. WIEGEL \\ Department of Applied Physics, Twente University of Technology, Enschede, The Netherlands
}

and

\section{J.PJ. MICHELS}

Von der Waals Laboratory, University of Amsterdam, Amsterdam, The Netherlands

Received 16 February 1976

\begin{abstract}
Using a simple functional relation the distribution of the times of free flight in a classical gas of hard spheres is shown to depend only on two specific combinations of the molecular and the:mociynamic parameter. This distribution has been calculated with molecular dynamics for tinree values of the density. The behaviour of the function is found to be qualitatively similar to its form in the low density limit, even for densities for which the system is far into the crystalline phase.
\end{abstract}

Let $p(\tau) \mathrm{d} \tau$ denote the probability over an equilibrium ensemble that the time of free flight of a hard sphere will be between $\tau$ and $\tau+\mathrm{d} \tau$. Let $p(v, \tau) \mathrm{d} \tau \mathrm{de}$ note the probability of the same event taken only over those particles in the equilibrium ensemble which have instantaneous velocity $v$. The two functions are related by:

$p(\tau)=\int f^{e q}(v) p(v, \tau) \mathrm{d}^{3} \mathrm{v}$,

where

$f^{e q}(v)=(2 \pi k T / m)^{-3 / 2} \exp \left(-m v^{2} / 2 k T\right)$

denotes the Maxwell-Boltzmann distribution, $m$ the mass of a sphere, $T$ the temperature and $k$ Boltzmann's constant.

The function $p(v, \tau)$ is physically significant because this function determines some of the transport coefficients of the hard sphere gas. For example, by calculating the transfer of momentum through a fictitious surface in a hard sphere gas which is in a macroscopic state of uniform shear one finds that the viscosity is rigorously given by:

$\eta=\frac{\pi}{3} \pi m \rho \int_{0}^{\infty} \mathrm{d} u v^{4} f^{\mathrm{eq}}(v) \int_{0}^{\infty} p(u, \tau) \tau \mathrm{d} \tau$, where $\rho$ denotes the number density. In the present note we shall consider the free flight time distribution for its own sake. Before doing so we would like to recall how this function can be calculated explicitly for small densities (most textbooks on kinetic theory discuss this problem). In contrast to the situation at higher densities molecular chaos holds at low densities. This straightforwardly leads to the low density form (indicated with subscripts 0 ):

$$
\begin{aligned}
& p_{0}(v, \tau)=n(v) \mathrm{e}^{-n(v) \tau}, \\
& n(v)=\pi \rho \sigma^{2}(m / 2 \pi k T)^{3 / 2} \\
& \quad \times \int\left|v^{\prime}-v\right| \exp \left[-m\left(v^{\prime}\right)^{2} / 2 k T\right] \mathrm{d}^{3} v^{\prime},
\end{aligned}
$$

where $\sigma$ denotes the diameter of the hard spheres. Substituting into (1) the low density limit of the free flight time distribution is found to have the form:

$p_{0}(\tau)=\rho \sigma^{2}(k T / m)^{1 / 2} P\left(\rho \sigma^{2}(k T / m)^{l / 2} \tau\right)$,

where

$$
\begin{aligned}
& P(\theta) \equiv \frac{1}{3} \int_{0}^{\infty} y^{2} \phi(y) \exp \left[-\frac{1}{2} y^{2}-\left(\frac{1}{18} \pi\right)^{1 / 2} \theta \phi(y)\right], \\
& \phi(y) \equiv \int_{0}^{\infty}(x / y)\left(|x+y|^{3}-|x-y|^{3}\right) \exp \left(-\frac{1}{2} x^{2}\right) d x .
\end{aligned}
$$




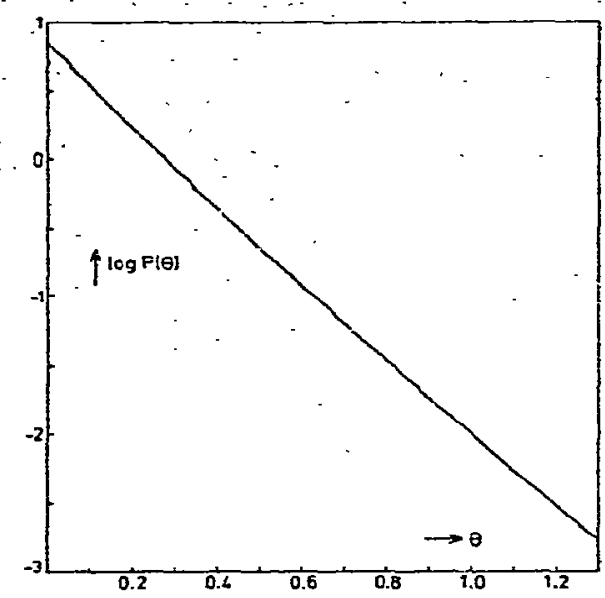

Fig. 1. Low density limit of the free flight time distribution. For notation compare eq. (5).

The function $P(\theta)$ has been calculated numerically and the results are given in a logarithmic plot (fig. 1). Quantitatively $P$ can be approximated by an exponen tial

$P(\theta) \approx 7.09 \exp (-7.090)$.

For arbitrary densities the function $p(v, \tau)$ obeys a simple functional relation which is found as follows. Imagine the gas in thermal equilibrium at a temperature $T$ and now (at zero time) instantaneously increase all the velocities by a factor $\alpha$. Then, for positive times, the gas is again in thermal equilibrium, but now at a temperature $\alpha^{2} T$. This is the case because the trajectories of the hard spheres are unchanged; the particles will follow their trajectories faster by a factor $\alpha$. Therefore, the sequence of collisions between spheres will be the same, but all the free flight times have to be multiplied by $\alpha^{-1}$. This gives:

$p(u, \tau, T)=\alpha^{-1} p\left(\alpha u, \alpha^{-1} \tau, \alpha^{2} T\right)$.

Taking $\alpha=\tau$ one finds that $p$ has the form $T^{1 / 2} G_{1}(u \tau$, $\left.T \tau^{2}\right)$. Now $k, T$ and $m$ enter this problem only in the combination $k T / m$, which appears in the equilibrium distribution (2), so $p$ must have the form $(k T / m)^{1 / 2} \times$ $G_{2}\left(u \tau, \tau(k T / m)^{1 / 2}\right)$. As far as the dependence on $\rho$ and $\sigma$ is concerned one can always write:

$p(v, \tau)=\rho \sigma^{2}(k T / m)^{1 / 2} G_{3}\left(v \tau, \tau(k T i m)^{1 / 2}, \rho \sigma^{2}, \rho \sigma^{3}\right)$, where $G_{3}$ denotes a-dinensionless function of the
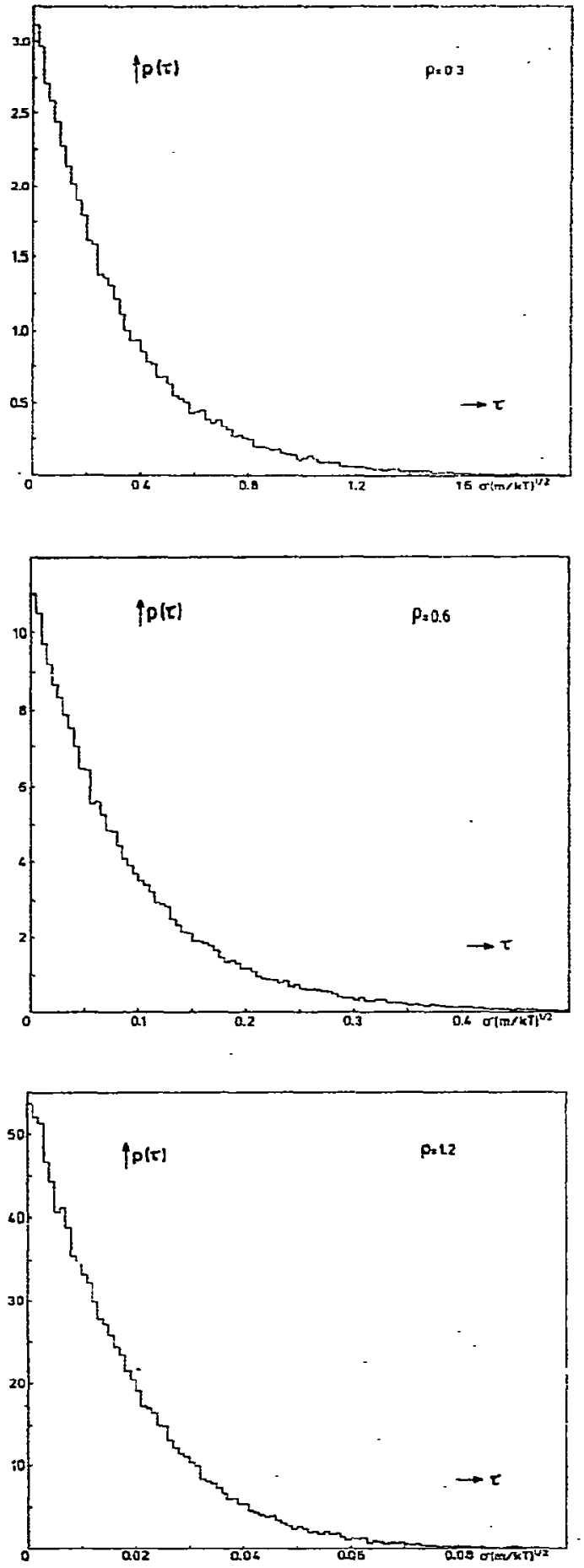

Fig. 2. Molecular dynamics calculation of the free flight time distribution for densities; (a) $\rho \sigma^{3}=0.3$; (b) $\rho \sigma^{3}=0.6$; (c) $\rho \sigma^{3}$ $=1.2$. The horizontal axis gives the value of $(\tau / \sigma)(k T / m)^{1 / 2}$. 
four variables indicated and where $v \equiv|v|$. One easily shows that only three dimensionless combinations of these variables exist, so that finally:

$$
\begin{aligned}
& p(v, \tau)=\rho \sigma^{2}(k T / m)^{1 / 2} \\
& \quad \times G\left(m v^{2} / k T, \rho \sigma^{2}(k T / m)^{1 / 2} \tau, \rho \sigma^{3}\right) .
\end{aligned}
$$

Substituting this formula into (1) the "scaled" form of the free flight time distribution is found:

$p(\tau)=\rho \sigma^{2}(k T / m)^{1 / 2} F\left(\rho \sigma^{2}(k T / m)^{1 / 2} \tau, \rho \sigma^{3}\right)$.

The limit of $F$ for $\rho \sigma^{3} \rightarrow 0$ has been given in eq. (5).

The distribution $p(\tau)$ was calculated numerically with molecular dynamics, for 500 hard spheres in a cubic volume. The densities were $\rho \sigma^{3}=0.3,0.6$ and 1.2. The calculation was done on a CDC 6500 electronic computer; the total number of collisions was 32581,41892 and 35228 . The results are given in figs. $2 a, 2 b$ and $2 c$.

It is obvious from these figures that, apart from a gradual shift of $p(\tau)$ towards shorter flight times, the qualitative behaviour of the distribution is similar to the low density limit (5). This feature is the more remarkable because a phase transition in the hard sphere fluid is found [1] at derisities $\rho \sigma^{3} \approx 0.9$. From the equation of state, and by observing the motion of the particles, Alder and Wainwright conclude that . this phenomenon is of the nature of a fluid-solid transition. Thus, from this point of view, for a density $\rho \sigma^{3}=1.2$ the system is far into the crystalline phase. Nevertheless it appears from our results that on a time scale of the order of the mean free flight time the motions of the particles are still as irregular as in the fluid phase.

If the function $p(v, T)$ behaves in a similar way, then eq. (3) leads to a finite value for the shear viscosity of the "crystalline" phase. However, it is conceivable that for large densities $f p(u, \tau) \tau \mathrm{d} \tau$ diverges; on the other hand a system consisting of a few hundred particles only would probably be too small to reproduce this effect.

\section{Reference}

[1] T. Wainwight and B.J. Alder, Nuovo Cimento 9.Suppl. $10(1958) 116$. 